Review

\title{
RNAs - Physical and functional modulators of chromatin reader proteins ${ }^{2}$
}

\author{
Kyoko Hiragami-Hamada, Wolfgang Fischle* \\ Laboratory of Chromatin Biochemistry, Max Planck Institute for Biophysical Chemistry, Am Fassberg 11, 37077 Göttingen, Germany
}

\section{A R T I C L E I N F O}

\section{Article history:}

Received 3 January 2014

Received in revised form 21 March 2014

Accepted 24 March 2014

Available online 2 April 2014

\section{Keywords:}

Epigenetics

Histone modification

Chromodomain protein

Non-coding RNA

Nascent transcript

\begin{abstract}
A B S T R A C T
The regulatory role of histone modifications with respect to the structure and function of chromatin is well known. Proteins and protein complexes establishing, erasing and binding these marks have been extensively studied. RNAs have only recently entered the picture of epigenetic regulation with the discovery of a vast number of long non-coding RNAs. Fast growing evidence suggests that such RNAs influence all aspects of histone modification biology. Here, we focus exclusively on the emerging functional interplay between RNAs and proteins that bind histone modifications. We discuss recent findings of reciprocally positive and negative regulations as well as summarize the current insights into the molecular mechanism directing these interactions. This article is part of a Special Issue entitled: Molecular mechanisms of histone modification function.
\end{abstract}

(c) 2014 Published by Elsevier B.V.

\section{Introduction}

Eukaryotic genomes exist in a complex of DNA, proteins and RNA called chromatin. The basic unit of chromatin is the nucleosome core particle, which consists of 147 bp DNA wrapped around an octameric center of histone proteins (two copies of each of the core histones $\mathrm{H} 2 \mathrm{~A}, \mathrm{H} 2 \mathrm{~B}, \mathrm{H} 3$ and H4) [1]. Each individual histone can be subjected to various covalent chemical modifications by specific chromatin modifier proteins ('writers' of histone modifications). These modifications include methylation, acetylation, phosphorylation, and ubiquitylation marks on multiple residues. The modifications are generally reversible and can be removed by the enzymatic action of 'erasers' of histone modifications (reviewed in [2-5]). Combinations of histone marks in the context of histone proteins, nucleosomes or defined regions of chromatin are thought to generate epigenetic codes. These are recognized and bound by one or more 'readers' of histone modifications to exert downstream effects on chromatin structure and/or gene activities (reviewed in $[3,6]$ ).

Interaction of reader proteins with their matching histone modifications is rather weak - the dissociation constants $(\mathrm{Kd})$ of most isolated binding pairs being in the micromolar range [7-10]. It is therefore puzzling, how these chromatin factors find their target genomic sites in the nucleus. Pure mass action and diffusion-controlled processes

\footnotetext{
This article is part of a Special Issue entitled: Molecular mechanisms of histone modification function.

* Corresponding author. Tel.: +495512011340.

E-mail address: wfischl@gwdg.de (W. Fischle).
}

are unlikely sufficient to explain the strong codistribution of marks and readers observed in genome wide studies. Biochemical extraction procedures (e.g. by salt or detergent) are indeed indicative of a relatively strong association of the readers with chromatin in cells [10-12]. Obviously other chromatin components mediate and stabilize the interaction. While multiprotein complexes containing different chromatin binding functionalities have long been recognized in this context, RNAs have only recently entered the picture.

While the first RNA-chromatin protein interaction was described in Drosophila more than ten years ago [13], only recent advances in methodologies (for example, UV-mediated crosslinking of RNA and proteins and immunoprecipitation (so-called 'PAR-CLIP') in combination with RNA-sequencing and/or mass spectrometry [14]) demonstrated the idea of prevalent interactions between coding and/or non-coding (nc) RNAs and a multitude of chromatin-associated proteins. Many of these are modifying enzymes including writers of histone modifications (Ezh2, Mll1, TIP5, ESET, SETD8, Suv39h1, G9a, DNMT3b) and erasers of the modifications (Jarid1B, Jarid1C, LSD1, HDACs) (reviewed in [15], [16-18]). Their interaction with specific RNAs, especially with long non-coding RNAs (IncRNAs), has been implicated in recruitment of these enzymes to their target genomic sites in cis and/or in trans thereby mediating epigenetic regulation of key genes in development and carcinogenesis. Several recent overview articles have summarized the biology of interactions between RNAs and chromatin modifying enzymes [15,19-21]. However, RNA-binding activities of a subset of 'reader' proteins of chromatin modifications have also been described in different experimental systems $[7,13,16,22-25]$. Here we will summarize the current knowledge on these interactions with particular emphasis on chromodomain proteins, whose RNA-binding activities have been 
investigated in depth. Possible physical and functional properties of RNA-chromatin reader interactions are discussed.

\section{Chromodomain proteins binding RNA}

Chromodomains are highly conserved structural motifs of $\sim 40$ 60 amino acids found in several chromatin associated proteins. It features an $\mathrm{N}$-terminal three-stranded anti-parallel $\beta$-sheet that folds against an $\mathrm{N}$-terminal $\alpha$-helix (Fig. 1A). This folding results in formation of an aromatic cage constituted of three aromatic amino acids that are necessary for recognizing methylated lysine residues in histones as well as non-histone proteins (reviewed in [26]). Chromodomains were first described in Drosophila modifiers of variegation proteins that are implicated in changing chromatin structure to the condensed morphology of heterochromatin. While different chromodomain proteins have been extensively studied in the context of binding histone methyl-lysine residues, chromodomain proteins of the Polycomb (Pc) protein family as well as homologues of Heterochromatin Protein 1 (HP1) have recently been recognized to also bind RNA.

Pc proteins are classically known to bind to methyl-H3K27 marks via their chromodomain. These mediate transcriptional silencing of facultative heterochromatin, including the mammalian inactive $\mathrm{X}$ chromosome, imprinted genes and developmentally regulated genes (reviewed in [27]). Several Pc proteins are present in mouse and human cells; Cbx2, Cbx4, Cbx6, Cbx7 and Cbx8. HP1 is a structural component of constitutive heterochromatin (found at pericentromeres and telomeres) and plays key roles in heterochromatin-mediated gene silencing. This factor is evolutionarily conserved from yeast to human. In mammals there are three HP1 proteins, HP1 $\alpha, \mathrm{HP} 1 \beta$ and $\mathrm{HP} 1 \gamma$. All
HP1 homologues consist of two globular domains; the chromodomain and chromoshadow domain that are connected by a less structured hinge region (Fig. 1B) (reviewed in [28-30]). The HP1 chromodomain binds to methyl-H3K9 [31], whereas the chromoshadow domain mediates self-dimerization and interaction with a wide range of other chromatin-associated proteins [32].

Although their amino acid sequences are fairly similar (Fig. 1A), only the Pc chromodomains possess RNA-binding capacity [7,23-25,33,34]. Besides Cbx2, all other Pc chromodomains showed RNA-binding activity in electrophoretic mobility shift assay (EMSA) using fragments of in vitro transcribed cyclin E RNA as bait [7]. Binding strength for ssRNA was considerably higher than that for dsRNA or dsDNA. Interestingly, the chromodomains seem to preferentially recognize a secondary structure of ssRNA as interaction was abolished when RNA was heatdenatured prior to the binding assay. In agreement, it was shown that interaction between Cbx7 and ANRIL, an IncRNA, occurs via recognition of a stem-loop structure. Several positively charged amino acids (R17, $\mathrm{K} 31$; Fig. 1A, indicated in pink) in the chromodomain were found to be important in mediating the interaction [33]. Mutations that disrupt the interaction between Cbx7 and methyl-H3K27 did not affect the RNA-binding activity of the protein, indicating that these two binding events are independent of each other [7,33]. Interestingly, these two basic amino acid residues are conserved in $\mathrm{Cbx} 4, \mathrm{Cbx} 6$ and $\mathrm{Cbx} 8$. Thus, they may also contribute to RNA-binding of these proteins (Fig. 1A).

In contrast to Pc proteins, homologues of HP1 use clusters of basic amino acid residues in the hinge region to interact with RNA (Fig. 1B, all $\mathrm{K} / \mathrm{R}$ are indicated in red) $[23,25,34]$. According to competitive EMSA, mammalian HP1 proteins also bind more preferentially to ssRNA than to ss/dsDNA [23].
A

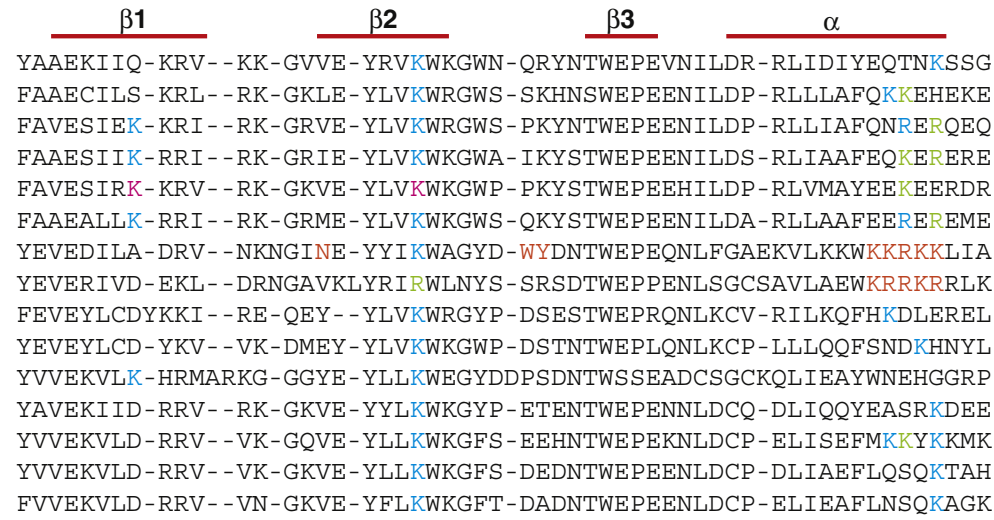

HP1 homologues/isoforms

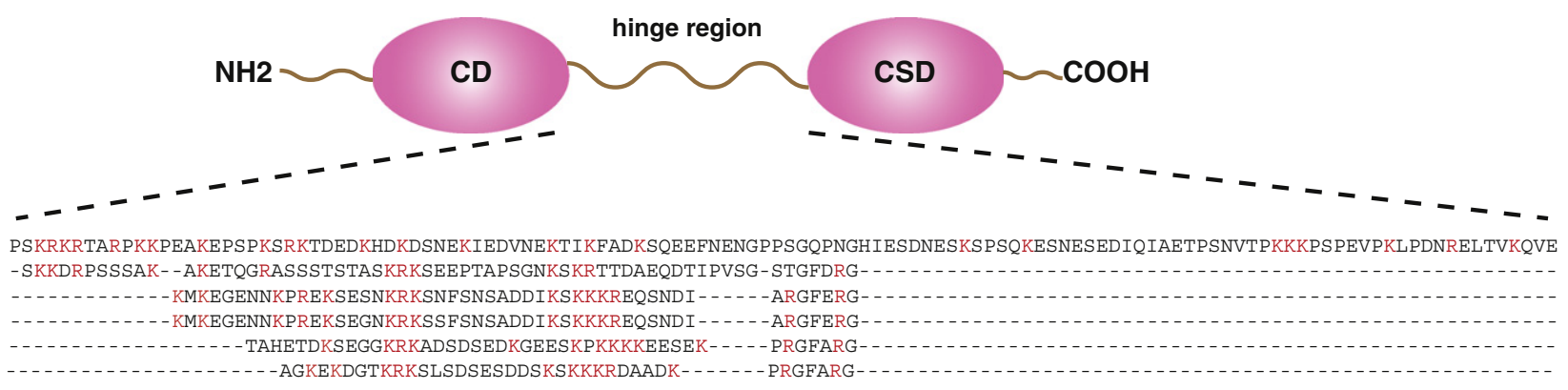

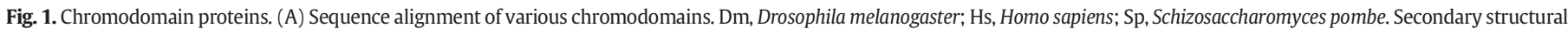

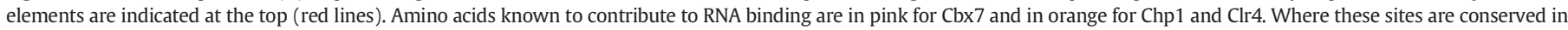

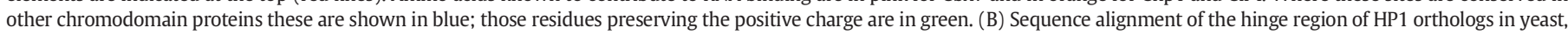
Drosophila (Dm), mouse (Mm) and human (Hs). Basic amino acids in the region are marked in red. 
2.1. RNAs function in recruitment of chromodomain proteins to large nuclear domains

Besides the in vitro binding studies, a first cellular link between chromodomain proteins and RNA was established by immunofluorescence-based assays, in which cells were first permeabilized and treated with RNase A before fixation and immunostaining [22]. Although this approach is unable to discriminate direct and indirect interactions, these studies revealed that RNA components contribute to targeting of the chromodomain proteins to large heterochromatic domains in mammalian cells. Several groups have shown that heterochromatic localization of HP1 $\alpha$ and/or HP1 $\gamma$ is RNase A-sensitive in mouse fibroblasts $[22,23]$. Consistently, the pericentromeric localization of HP1 $\alpha$ was restored upon addition of total or nuclear RNA from the same cellular source to RNase A-treated samples but not upon addition of tRNA or bacterial RNA, suggesting some degree of specificity of the interaction [22]. Later, direct binding of mouse HP1 $\alpha$ to transcripts from the major satellite repeats was demonstrated [34]. Interaction of HP1 $\alpha$ with these RNAs appears crucial for de novo recruitment as well as anchoring of the factor to heterochromatic foci (Fig. 2A). The RNAHP1 interaction is strongly enhanced by sumoylation of the protein thereby directing HP1 $\alpha$ recruitment to heterochromatic foci even in absence of the trimethyl-H3K9 mark [34].

Physiological importance of the interaction of HP1 with the RNA derived from the major satellite repeats has been demonstrated during epithelial to mesenchymal transition (EMT). Several transcription factors such as Snail1, Pax3 or Pax9 and an amino oxidase, LOXL2 regulate transcription from these loci $[35,36]$. Suppression of expression by cooperative action of Snail1 and LOXL2 causes transient release of HP1 $\alpha$ from heterochromatic foci, a process which is presumably necessary for heterochromatin reorganization to complete the EMT [36].

In the case of Pc proteins, it was found that targeting of EGFP-Cbx7 to the inactive $\mathrm{X}$ chromosome is also RNase A-sensitive in differentiating mouse ES cells [7]. Expression of Xist lncRNA is the initiating step of X inactivation in female mammalian cells. However, interaction of Pc proteins with Xist is highly controversial (for comprehensive review, see [37]). The observation nevertheless implies that RNA components contribute to anchoring and/or de novo recruitment of $\mathrm{Cbx} 7$ to the facultative heterochromatin of the inactive $\mathrm{X}$ chromosome. This effect might not be direct, but could be mediated by targeting more prominent Xist binding proteins such as components of Polycomb Repressor Complex 2 (PRC2), Ezh and Suz12, that establish methyl-H3K27 marks for Cbx7 binding.

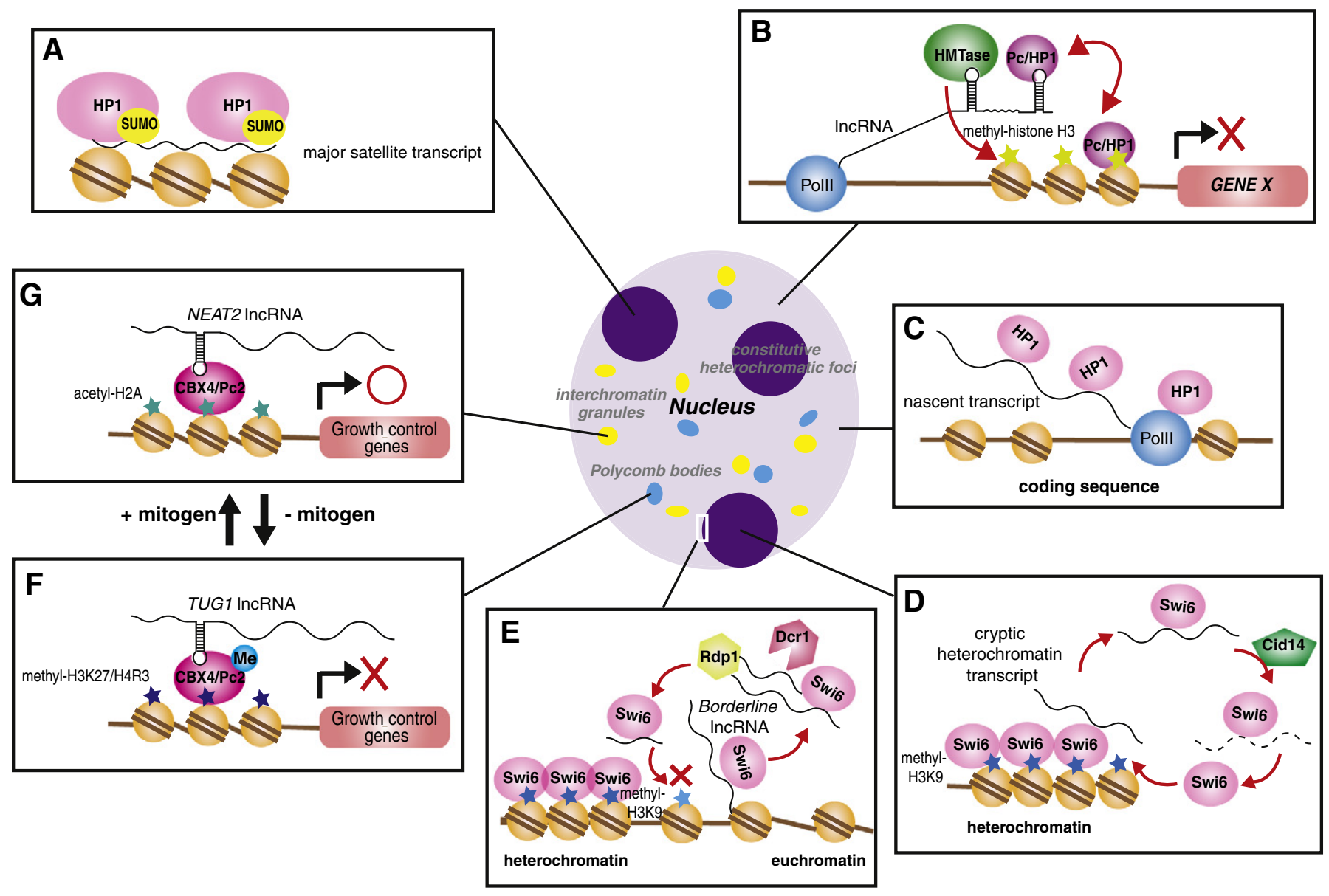

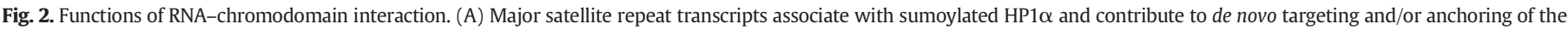

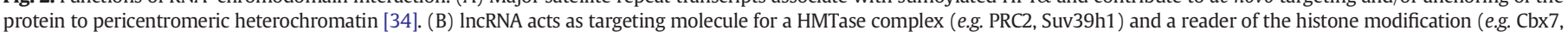

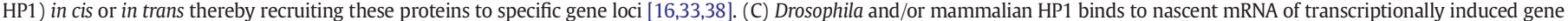

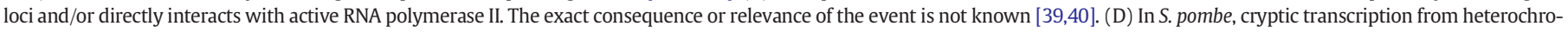

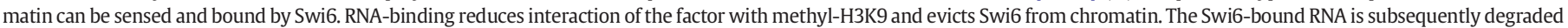

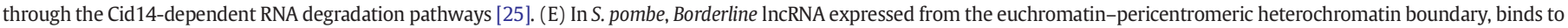

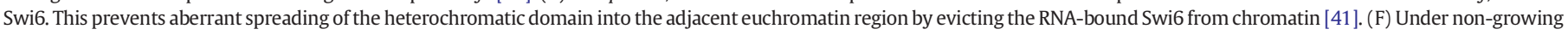

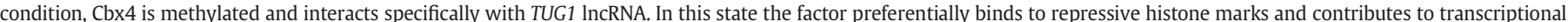

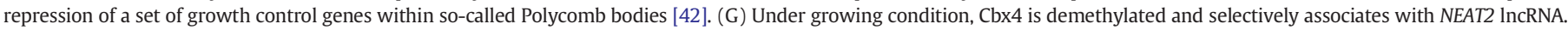

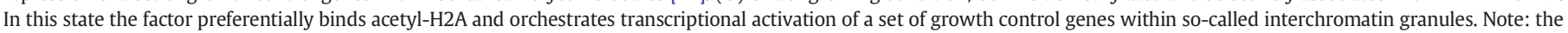
processes outlined in (F) and $(G)$ are dynamic and reversible depending on the presence/absence of mitogenic stimuli [42]. 


\subsection{RNAs targeting chromodomain proteins to specific loci}

More recent studies suggest that ncRNAs are involved in targeting of chromodomain proteins not only to large chromatin regions but also to individual gene loci (Fig. 2B). Cbx7 binds to ANRIL IncRNA, whose expression leads to silencing of INK4b/ARF/INK4a tumor suppressor loci in prostate cancer cells [33]. Chromatin immunoprecipitation (ChIP) experiments of Ezh2, Cbx7 and H3K27me3 in WT and ANRIL knock-down cells indicated that the IncRNA is required for recruitment of Ezh2 and Cbx7 to the tumor suppressor loci. However, disruption of the RNA-binding activity of Cbx7 led to only minor effects on colony formation/proliferative capacity of mouse embryonic fibroblasts. Hardly any effects on reactivation of the target tumor suppressor genes were seen. Conversely, overexpression of a Cbx7 mutant that is defective in binding methylH3K27 had striking effects on cell proliferation and target gene derepression. Hence, IncRNA-Cbx7 interaction may not be crucial in regulating these events but may be involved in fine-tuning of the effect mediated primarily by methyl-H3K27-Cbx7 interaction.

Similarly, RNA-mediated recruitment of HP1 $\gamma$ to specific gene loci has been described in human cells [38]. Together with progesterone receptor (PR) and LSD1, HP1 $\gamma$ associates with a specific RNA known as steroid receptor RNA activator (RNA SRA) to repress a large set of hormone-inducible genes in the absence of ligand. In this case, the protein-RNA interaction is important in recruiting and anchoring the protein complex at its genomic target sites. Furthermore, using a candidate approach, interactions between several readers, writers and erasers of chromatin modifications and IncRNAs were described in mouse ES cells [16]. For example, HP1 $\beta$ and HP1 $\gamma$ were found to bind distinct sets of IncRNAs, in some cases putatively together with a methyl-H3K9 writer, ESET or Suv39h1. The studies suggest coordinated recruitment of a writer and a reader of histone modification by lncRNAs to their target loci for rapid gene shutdown.

In contrast to the above examples of targeting to silenced regions of the genome, recruitment of HP1 homologues to transcriptionally active loci has also been reported (Fig. 2C). In Drosophila RNase A-sensitive localization of HP1 was observed at transcriptionally induced heat shock protein loci [39]. This implied that recruitment of HP1 to the heat shock puffs is at least partially dependent on nascent transcripts and possible interaction between HP1 and the nascent RNA. Although neither RNase A sensitivity nor direct nascent RNA-HP1 interaction was tested, mammalian HP1 $\gamma$ and H3K9me3 marks were also shown to be enriched at transcribed/coding regions of transcriptionally induced genes including the IL2 locus in mouse myeloid and lymphoid cells [40]. These binding events might be involved in resetting the transcriptional cycle of initiation-elongation-termination [39]. Yet, the exact molecular and biological consequences of the HP1-nascent mRNA interaction remain enigmatic.

At least in metazoans association of chromodomain proteins with RNA seems to contribute to proper targeting of HP1 or Pc proteins to heterochromatic as well as euchromatic target sites (Fig. 2A-C). Cobinding of these readers and chromatin-modifying enzymes to IncRNAs raise the possibility that the latter act as molecular scaffolds for the repressor complexes thereby promoting coordinated recruitment to target loci (Fig. 2B). While this is an appealing idea, it needs to be noted that the resolution of the current experiments is not high enough to clarify whether the identified protein-RNA interactions are direct or not. This applies to immunofluorescence-based techniques as well as crosslinking of RNAs and proteins for immunoprecipitation, since the generally used formaldehyde unspecifically connects not only RNA with proteins but also proteins with proteins. Direct interaction of the chromodomain proteins and their partner writer protein with RNAs needs to be confirmed in vivo by alternative methods such as PAR-CLIP, which mediates more specific crosslinking and is limited to capture only very short-range RNAprotein interactions.

\subsection{RNAs evicting chromodomain proteins}

Work on metazoan HP1 and Pc proteins has implied an attracting mode of RNAs in binding of chromodomain proteins to chromatin. However, recent studies on the HP1 orthologue in Schizosaccharomyces pombe (S.pombe), Swi6, challenged this paradigm by indicating that RNAs can also repel chromodomain proteins from their targets (Fig. 2D, E) [25,41]. Like metazoan HP1, Swi6 binds to RNA through its hinge region that is much longer and contains more basic amino acids (Fig. 1B). NMR analysis of Swi6 in the absence or presence of synthetic 20-mer GFP RNA revealed that addition of RNA changes the chemical environment not only of amino acids in the hinge region but also of those located in the chromodomain and N-terminal region [25]. Obviously, interaction with RNA modulates the conformation of the chromodomain, which has negative effects on Swi6 binding to methyl-H3K9. Such dissociation or eviction of RNA-bound Swi6 from pericentromeric heterochromatin appears to be important in trapping and priming heterochromatic RNAs for Cid14-dependent degradation through the RNA exosome and/ or RNAi pathways (Fig. 2D) [25]. Furthermore, binding of Swi6 to a IncRNA called Borderline, which is expressed from the boundary region between pericentromeric heterochromatin and neighboring euchromatin, prevents aberrant spreading of the heterochromatic domain (marked by Swi6 and H3K9me2) into adjacent euchromatic regions (Fig. 2E) [41]. In this case, Borderline RNA appears to act as an evictor of HP1. It is subsequently processed into non-canonical small RNAs, which cannot be loaded onto a part of the RNAi machinery, Ago1. These small ncRNAs are therefore not fed back into the RNAi pathway for propagation of heterochromatin formation. Although it is unclear if such an RNA-mediated conformational change of chromodomain structure and/or an evictor effect of RNA apply to metazoan HP1s, these findings indicate that RNAs can generally exert positive as well as negative effects on the chromatin binding of these factors.

\subsection{RNAs as master regulators of chromodomain protein function}

Besides RNAs as targeting molecules for chromodomain proteins, analysis of $\mathrm{Cbx} 4$ biology implies multi-layered regulation by interaction with RNA (Fig. 2F, G) [42]. It was shown that Cbx4 directly binds to IncRNAs, TUG1 and NEAT2 in human cell lines. The specificity of the Pc protein for these RNAs is regulated by Suv39h1/KDM4C-mediated methylation/demethylation of a single residue of the protein, K191, which lies outside of the chromodomain. Methylated Cbx4 predominantly binds TUG1 and localizes to repressive Polycomb bodies (PcBs). In contrast, unmethylated $\mathrm{Cbx} 4$ preferentially interacts with NEAT2 and is found in transcriptionally permissive interchromatin granules (ICGs). The consequences of selective binding of $\mathrm{Cbx} 4$ to the different IncRNAs are striking. It results in relocation of $\mathrm{Cbx} 4$-bound growth control genes from PcBs to ICGs concomitant with orchestrated transcriptional activation in response to mitotic stimuli. Obviously, the IncRNAs act as structural scaffolds or chaperons to anchor/relocate the Pc protein complex and associated genomic regions within or between specific subnuclear compartments. Moreover, differential binding of the two lncRNAs to Cbx4 results in changes in the specificity of Cbx4 towards histone modifications (see Section 3 for details). Altogether, these observations indicate that the distinct lncRNAs influence multiple properties of $\mathrm{Cbx} 4$, thereby acting as master regulators of its functions. The exact molecular mechanisms of this intriguing pathway still need to be unraveled. Also, it will have to be seen whether other factors are controlled in their function in a similar manner.

\section{RNA and histone modification binding can influence each other}

How does the functional interplay of RNAs and histone modification readers work on a molecular level? An intriguing emerging concept is direct modulation of binding properties. As discussed above, it was recently shown that RNA interaction reduces the methyl-H3K9 binding 
activity of Swi6 [25]. Even more intriguingly, Yang et al. demonstrated a switch-like behavior of $\mathrm{Cbx} 4$ histone modification binding directed by IncRNAs [42]. Unlike most Pc proteins the chromodomain of this factor shows highest specificity for H3K9me3 when not bound to RNAs [7]. Interaction with TUG1 directs preferential recognition of other repressive histone modifications such as symmetrically methylated H4R3me2 and H3K27me2. In contrast, association with NEAT2 reduces $\mathrm{Cbx} 4$ binding to H3K9me3 resulting in binding to transcriptionally active marks including acetyl-H2A. Such a switch in specificity is expected to have profound effects on the coordinated regulation of a set of growth control genes [42].

Direct interplay between methyl-H3K9 binding and RNA-binding activities of chromodomains has also been demonstrated for the Chp1 and $\mathrm{Clr} 4$ proteins in S. pombe [24]. Interestingly, this organism does not have any Pc like factors. The chromodomains of both, Chp1 and Clr4 specifically interact with methyl-H3K9 [43-45]. While Chp1 is a part of the RNAi-induced transcriptional silencing (RITS) complex whose core subunits consist of Argonaute, Tas 3 and $\mathrm{Chp} 1, \mathrm{Clr} 4$ is a H3K9-specific methyltransferase (HMTase). Both proteins play pivotal roles in the RNAi pathway and in heterochromatin-mediated gene silencing in yeast (reviewed in [30], [43,46]). For both Chp1 and Clr4, the interaction with RNA is partly mediated by a stretch of 5-6 basic amino acids located at the C-terminus of the $\alpha$-helix within their chromodomains (Fig. 1A, indicated in orange) [24]. In the case of Chp1, aromatic amino acids (WY) and an asparagine (N) of the chromodomain also contribute to RNA binding (Fig. 1A, indicated in orange). These residues are absent in the Clr4 chromodomain, which binds to RNA only in a context dependent manner (see below).

For both factors it was shown that interaction with methyl-H3K9 enhances the RNA-binding activity of the chromodomain. While the effect is subtle for Chp1 it is more striking for Clr4. The chromodomain of $\mathrm{Clr} 4$ alone does not bind to RNAs as tested by EMSA. However, in the presence of $0.5-1 \times$ molar excess of methyl-H3K9me3, RNA-binding capacity was observed [24]. The findings indicate that the binding of the chromodomains of Chp1 and Clr4 to their target histone mark leads to conformational changes exposing (additional) RNA-interacting surfaces. The interplay between the two distinct binding events has biological relevance as there are clear additive defects on the heterochromatic silencing and siRNA synthesis upon combinatory mutations that block both H3K9me-binding and RNA-binding of Chp1 chromodomain [24].

The current examples indicate that RNA binding of the chromodomain proteins can influence their interaction with methyl-histone marks and vice versa. Likely, engagement of the chromodomain with RNA/methylhistone marks induces significant allosteric changes in the globular domain, affecting the other or subsequent binding events. Comparison of the structures of free, RNA or methyl-histone bound as well as RNA and methyl-histone bound chromodomains will be necessary to give further insights into such conformational changes and help to identify the 'hidden' and/or true RNA-interacting surface(s) of the chromodomains.

\section{Interaction of RNA with histone modification readers: promiscuity $v s$. specificity}

The RNA binding of both Pc proteins and HP1 appears to involve electrostatic interactions between the positively charged basic amino acids and the negatively charged sugar-phosphate backbone of RNAs. Obviously, there are many types of RNAs in a cell's nucleus: nascent/ primary transcripts, ribosomal RNAs, (peri-)centromeric transcripts, small ncRNAs such as small nucleolar RNAs as well as various lncRNAs (anti-sense transcripts, intronic IncRNAs, circular RNAs, enhancer IncRNAs, etc., reviewed in [47], [48-50]). So, is there any specificity in the binding of histone modification readers with RNA?

The answer to the question may be both 'Yes' and 'No'. While some degree of specificity for RNA-binding activities has been suggested [7, $22,23,42]$, chromodomains and/or other chromatin proteins may also bind to RNAs in a more promiscuous manner. Hints in this direction come from a histone modification writer, Ezh2 that methylates H3K27 and is a bona fide RNA-binding chromatin protein. Physiologically relevant interaction of Ezh2 with different specific IncRNAs has been described. However, these findings were based on candidate approaches where only selected RNAs were analyzed (reviewed in [15,19-21]). More recently, unbiased analysis of Ezh2-bound RNA species, however, identified very diverse sets of RNAs including nascent transcripts at transcriptionally active gene loci $[51,52]$. Surprisingly, the interaction with nascent transcripts does not result in local H3K27 methylation nor in Polycomb mediated gene silencing, suggesting that a nascent transcript may be acting as an inhibitor/evictor for PRC2 or as a decoy for 'functional' RNA-protein interaction. Similar to HP1, interaction between Ezh2 and RNAs seems to involve a domain containing a short stretch of basic amino acids [53]. While HP1 has longer and denser patches of K/Rs, short stretches of K/Rs are present in most nuclear proteins as part of nuclear localization signals. It needs therefore to be investigated whether unspecific RNA binding is a global phenomenon or applies only to some proteins containing higher charge density. Analysis of putative RNA binding of other readers but also writers and erasers of chromatin modifications in an unbiased manner will aid in this endeavor.

If there is general unspecific interaction, how do histone modification readers discriminate such events from specific RNA binding? Firstly, posttranslational modifications of the chromatin proteins may exert such a regulatory effect. As mentioned earlier, methylation and demethylation of $\mathrm{Cbx} 4$ establish a switch for differential binding of the protein to distinct lncRNAs [42]. It has been shown that the charged hinge region of mammalian HP1 can be adorned with various post-translational modifications including phosphorylation, methylation and sumoylation [34,54, 55]. As discussed above, sumoylation of lysine residues in the hinge region of mouse HP1 $\alpha$ controls its binding to major satellite transcripts [34]. Besides, phosphorylation of Ezh2 is known to enhance its binding to HOTAIR lncRNA [53]. Secondly, the histone modification binding proteins may use different interaction modes or surfaces for the promiscuous vs. specific RNA interaction. Pc proteins might use the chromodomain for more specific or functional RNA-interactions, whereas undiscriminating binding might be mediated by a tract of K/Rs located elsewhere (mostly found in C-terminal to the chromodomain). The three dimensional architecture of the chromodomains might physically restrict the type of RNAs the proteins can accommodate. Thirdly and as seen with the Clr4 and Chp1 proteins, actual engagement of the chromodomain with methyl-histone marks may alter the RNA binding activity and specificity. We note that the chromodomains of mouse and human HP1 $\alpha$ contain a stretch of K/R at the C-terminus of the $\alpha$-helix (Fig. 1A). It will be interesting to investigate whether binding of the HP1 $\alpha$ chromodomain to methyl-H3K9 can expose/create another RNA-interaction with different binding properties and specificities.

\section{Future perspectives}

While the analysis of interactions between histone modification readers and RNAs is still in its early stages, it is already clear that these can have different molecular properties and cause diverse functional effects. A big question is the specificity of these interactions. In most cases, candidate approaches have been taken and unbiased genetic screens or systematic biochemical approaches identifying RNA-binding chromatin proteins and/or the RNAs that bind particular histone modification readers have not been applied. Thus, only certain aspects of the biology of such interactions have been analyzed using a limited number of experimental and model systems. Also, the exact modes of binding need to be addressed from multiple angles including biophysical, structural and biochemical approaches. Additional input to this new field is coming from large-scale screens identifying the interactome of poly $(A)+$ and other RNA species [56-58]. We have no doubt that a much bigger and far more detailed picture of RNA-histone modification reader interactions will rapidly emerge in the coming years. 


\section{Acknowledgements}

We thank A. Stuetzer, M. Nikolov, R. Finn and Y. Ostwal for critical reading of the manuscript.

K.H.H. is supported by a Marie Curie Intra-European Fellowship for Career Development (IEF328577, FP7). Work in the laboratory of W.F. is funded by the Max Planck Society (MTNABICH0001).

\section{References}

[1] K. Luger, A.W. Mader, R.K. Richmond, D.F. Sargent, T.J. Richmond, Crystal structure of the nucleosome core particle at 2.8 A resolution, Nature 389 (1997) 251-260.

[2] C.H. Arrowsmith, C. Bountra, P.V. Fish, K. Lee, M. Schapira, Epigenetic protein families: a new frontier for drug discovery, Nat. Rev. Drug Discov. 11 (2012) 384-400.

[3] C.A. Musselman, M.E. Lalonde, J. Cote, T.G. Kutateladze, Perceiving the epigenetic landscape through histone readers, Nat. Struct. Mol. Biol. 19 (2012) 1218-1227.

[4] M.D. Shahbazian, M. Grunstein, Functions of site-specific histone acetylation and deacetylation, Annu. Rev. Biochem. 76 (2007) 75-100.

[5] N. Mosammaparast, Y. Shi, Reversal of histone methylation: biochemical and molecular mechanisms of histone demethylases, Annu. Rev. Biochem. 79 (2010) 155-179.

[6] D.J. Patel, Z. Wang, Readout of epigenetic modifications, Annu. Rev. Biochem. 82 (2013) 81-118.

[7] E. Bernstein, E.M. Duncan, O. Masui, J. Gil, E. Heard, C.D. Allis, Mouse polycomb proteins bind differentially to methylated histone $\mathrm{H} 3$ and RNA and are enriched in facultative heterochromatin, Mol. Cell. Biol. 26 (2006) 2560-2569.

[8] W. Fischle, Y. Wang, S.A. Jacobs, Y. Kim, C.D. Allis, S. Khorasanizadeh, Molecular basis for the discrimination of repressive methyl-lysine marks in histone H3 by Polycomb and HP1 chromodomains, Genes Dev. 17 (2003) 1870-1881.

[9] W. Fischle, B.S. Tseng, H.L. Dormann, B.M. Ueberheide, B.A. Garcia, J. Shabanowitz, D. F. Hunt, H. Funabiki, C.D. Allis, Regulation of HP1-chromatin binding by histone H3 methylation and phosphorylation, Nature 438 (2005) 1116-1122.

[10] R. Eskeland, A. Eberharter, A. Imhof, HP1 binding to chromatin methylated at H3K9 is enhanced by auxiliary factors, Mol. Cell. Biol. 27 (2007) 453-465.

[11] E. Meshorer, T. Misteli, Chromatin in pluripotent embryonic stem cells and differentiation, Nat. Rev. Mol. Cell Biol. 7 (2006) 540-546.

[12] M.P. Torrente, B.M. Zee, N.L. Young, R.C. Baliban, G. LeRoy, C.A. Floudas, S.B. Hake, B.A. Garcia, Proteomic interrogation of human chromatin, PLoS ONE 6 (2011) e24747.

[13] A. Akhtar, D. Zink, P.B. Becker, Chromodomains are protein-RNA interaction modules, Nature 407 (2000) 405-409.

[14] A. Castello, R. Horos, C. Strein, B. Fischer, K. Eichelbaum, L.M. Steinmetz, J. Krijgsveld, M.W. Hentze, System-wide identification of RNA-binding proteins by interactome capture, Nat. Protoc. 8 (2013) 491-500

[15] J.L. Rinn, H.Y. Chang, Genome regulation by long noncoding RNAs, Annu. Rev. Biochem. 81 (2012) 145-166.

[16] M. Guttman, J. Donaghey, B.W. Carey, M. Garber, J.K. Grenier, G. Munson, G. Young, A.B. Lucas, R. Ach, L. Bruhn, X. Yang, I. Amit, A. Meissner, A. Regev, J.L. Rinn, D.E. Root, E.S. Lander, lincRNAs act in the circuitry controlling pluripotency and differentiation, Nature 477 (2011) 295-300.

[17] C. Mayer, M. Neubert, I. Grummt, The structure of NoRC-associated RNA is crucial for targeting the chromatin remodelling complex NoRC to the nucleolus, EMBO Rep. 9 (2008) 774-780.

[18] Y. Zhou, K.M. Schmitz, C. Mayer, X. Yuan, A. Akhtar, I. Grummt, Reversible acetylation of the chromatin remodelling complex NoRC is required for non-coding RNA-dependent silencing, Nat. Cell Biol. 11 (2009) 1010-1016.

[19] P.J. Batista, H.Y. Chang, Long noncoding RNAs: cellular address codes in development and disease, Cell 152 (2013) 1298-1307.

[20] L.R. Sabin, M.J. Delas, G.J. Hannon, Dogma derailed: the many influences of RNA on the genome, Mol. Cell 49 (2013) 783-794.

[21] J.T. Kung, D. Colognori, J.T. Lee, Long noncoding RNAs: past, present, and future, Genetics 193 (2013) 651-669.

[22] C. Maison, D. Bailly, A.H. Peters, J.P. Quivy, D. Roche, A. Taddei, M. Lachner, T. Jenuwein, G. Almouzni, Higher-order structure in pericentric heterochromatin involves a distinct pattern of histone modification and an RNA component, Nat. Genet. 30 (2002) 329-334.

[23] C. Muchardt, M. Guilleme, J.S. Seeler, D. Trouche, A. Dejean, M. Yaniv, Coordinated methyl and RNA binding is required for heterochromatin localization of mammalian HP1alpha, EMBO Rep. 3 (2002) 975-981.

[24] M. Ishida, H. Shimojo, A. Hayashi, R. Kawaguchi, Y. Ohtani, K. Uegaki, Y. Nishimura, J. Nakayama, Intrinsic nucleic acid-binding activity of Chp1 chromodomain is required for heterochromatic gene silencing, Mol. Cell 47 (2012) 228-241.

[25] C. Keller, R. Adaixo, R. Stunnenberg, K.J. Woolcock, S. Hiller, M. Buhler, HP1(Swi6) mediates the recognition and destruction of heterochromatic RNA transcripts, Mol. Cell 47 (2012) 215-227.

[26] K.L. Yap, M.M. Zhou, Structure and mechanisms of lysine methylation recognition by the chromodomain in gene transcription, Biochemistry 50 (2011) 1966-1980.

[27] A. Sparmann, M. van Lohuizen, Polycomb silencers control cell fate, development and cancer, Nat. Rev. Cancer 6 (2006) 846-856.

[28] C. Maison, G. Almouzni, HP1 and the dynamics of heterochromatin maintenance, Nat. Rev. Mol. Cell Biol. 5 (2004) 296-304.

[29] K. Hiragami, R. Festenstein, Heterochromatin protein 1: a pervasive controlling influence, Cell Mol. Life Sci. 62 (2005) 2711-2726.

[30] S.I. Grewal, S. Jia, Heterochromatin revisited, Nat. Rev. Genet. 8 (2007) 35-46.
[31] M. Lachner, D. O'Carroll, S. Rea, K. Mechtler, T. Jenuwein, Methylation of histone H3 lysine 9 creates a binding site for HP1 proteins, Nature 410 (2001) 116-120.

[32] S.V. Brasher, B.O. Smith, R.H. Fogh, D. Nietlispach, A. Thiru, P.R. Nielsen, R.W Broadhurst, L.J. Ball, N.V. Murzina, E.D. Laue, The structure of mouse HP1 suggests a unique mode of single peptide recognition by the shadow chromo domain dimer, EMBO J. 19 (2000) 1587-1597.

[33] K.L. Yap, S. Li, A.M. Munoz-Cabello, S. Raguz, L. Zeng S. Mujtaba, J. Gil, M.J. Walsh, M. M. Zhou, Molecular interplay of the noncoding RNA ANRIL and methylated histone H3 lysine 27 by polycomb CBX7 in transcriptional silencing of INK4a, Mol. Cell 38 (2010) 662-674

[34] C. Maison, D. Bailly, D. Roche, R. Montes de Oca, A.V. Probst, I. Vassias, F. Dingli, B. Lombard, D. Loew, J.P. Quivy, G. Almouzni, SUMOylation promotes de novo targeting of HP1alpha to pericentric heterochromatin, Nat. Genet. 43 (2011) 220-227.

[35] A. Bulut-Karslioglu, V. Perrera, M. Scaranaro, I.A. de la Rosa-Velazquez, S. van de Nobelen, N. Shukeir, J. Popow, B. Gerle, S. Opravil, M. Pagani, S. Meidhof, T. Brabletz, T. Manke, M. Lachner, T. Jenuwein, A transcription factor-based mechanism for mouse heterochromatin formation, Nat. Struct. Mol. Biol. 19 (2012) 1023-1030.

[36] A. Millanes-Romero, N. Herranz, V. Perrera, A. Iturbide, J. Loubat-Casanovas, J. Gil, T. Jenuwein, A. Garcia de Herreros, S. Peiro, Regulation of heterochromatin transcription by Snail1/LOXL2 during epithelial-to-mesenchymal transition, Mol. Cell 52 (5) (2013) 746-757.

[37] N. Brockdorff, Noncoding RNA and Polycomb recruitment, RNA 19 (2013) 429-442.

[38] G.P. Vicent, A.S. Nacht, R. Zaurin, J. Font-Mateu, D. Soronellas, F. Le Dily, D. Reyes, M. Beato, Unliganded progesterone receptor-mediated targeting of an RNA-containing repressive complex silences a subset of hormone-inducible genes, Genes Dev. 27 (2013) 1179-1197.

[39] L. Piacentini, L. Fanti, M. Berloco, B. Perrini, S. Pimpinelli, Heterochromatin protein 1 (HP1) is associated with induced gene expression in Drosophila euchromatin, J. Cell Biol. 161 (2003) 707-714

[40] C.R. Vakoc, S.A. Mandat, B.A. Olenchock, G.A. Blobel, Histone H3 lysine 9 methylation and HP1gamma are associated with transcription elongation through mammalian chromatin, Mol. Cell 19 (2005) 381-391.

[41] C. Keller, R. Kulasegaran-Shylini, Y. Shimada, H.R. Hotz, M. Buhler, Noncoding RNAs prevent spreading of a repressive histone mark, Nat. Struct. Mol. Biol. 20 (2013) 994-1000.

[42] L. Yang, C. Lin, W. Liu, J. Zhang, K.A. Ohgi, J.D. Grinstein, P.C. Dorrestein, M.G. Rosenfeld, ncRNA- and Pc2 methylation-dependent gene relocation between nuclear structures mediates gene activation programs, Cell 147 (2011) 773-788.

[43] M. Sadaie, T. Iida, T. Urano, J. Nakayama, A chromodomain protein, Chp1, is required for the establishment of heterochromatin in fission yeast, EMBO J. 23 (2004) 3825-3835.

[44] T. Schalch, G. Job, V.J. Noffsinger, S. Shanker, C. Kuscu, L. Joshua-Tor, J.F. Partridge, High-affinity binding of Chp1 chromodomain to K9 methylated histone H3 is required to establish centromeric heterochromatin, Mol. Cell 34 (2009) 36-46

[45] S. Rea, F. Eisenhaber, D. O'Carroll, B.D. Strahl, Z.W. Sun, M. Schmid, S. Opravil, K. Mechtler, C.P. Ponting, C.D. Allis, T. Jenuwein, Regulation of chromatin structure by site-specific histone H3 methyltransferases, Nature 406 (2000) 593-599.

[46] J. Nakayama, J.C. Rice, B.D. Strahl, C.D. Allis, S.I. Grewal, Role of histone H3 lysine 9 methylation in epigenetic control of heterochromatin assembly, Science 292 (2001) 110-113.

[47] S. Guil, M. Esteller, Cis-acting noncoding RNAs: friends and foes, Nat. Struct. Mol Biol. 19 (2012) 1068-1075.

[48] S. Guil, M. Soler, A. Portela, J. Carrere, E. Fonalleras, A. Gomez, A. Villanueva, M Esteller, Intronic RNAs mediate EZH2 regulation of epigenetic targets, Nat. Struct. Mol. Biol. 19 (2012) 664-670.

[49] J. Salzman, C. Gawad, P.L. Wang, N. Lacayo, P.O. Brown, Circular RNAs are the predominant transcript isoform from hundreds of human genes in diverse cell types, PLoS ONE 7 (2012) e30733.

[50] Y. Zhang, X.O. Zhang, T. Chen, J.F. Xiang, Q.F. Yin, Y.H. Xing, S. Zhu, L. Yang, L.L. Chen, Circular intronic long noncoding RNAs, Mol. Cell 51 (2013) 792-806.

[51] C. Davidovich, L. Zheng, K.J. Goodrich, T.R. Cech, Promiscuous RNA binding by Polycomb repressive complex 2, Nat. Struct. Mol. Biol. 20 (2013) 1250-1257.

[52] S. Kaneko, J. Son, S.S. Shen, D. Reinberg, R. Bonasio, PRC2 binds active promoters and contacts nascent RNAs in embryonic stem cells, Nat. Struct. Mol. Biol. 20 (2013) 1258-1264.

[53] S. Kaneko, G. Li, J. Son, C.F. Xu, R. Margueron, T.A. Neubert, D. Reinberg, Phosphorylation of the PRC2 component Ezh2 is cell cycle-regulated and up-regulates its binding to ncRNA, Genes Dev. 24 (2010) 2615-2620.

[54] G. LeRoy, J.T. Weston, B.M. Zee, N.L. Young, M.D. Plazas-Mayorca, B.A. Garcia, Heterochromatin protein 1 is extensively decorated with histone code-like posttranslational modifications, Mol. Cell Proteomics 8 (2009) 2432-2442.

[55] K. Hiragami-Hamada, K. Shinmyozu, D. Hamada, Y. Tatsu, K. Uegaki, S. Fujiwara, ]. Nakayama, N-terminal phosphorylation of HP1\{alpha\} promotes its chromatin binding, Mol. Cell. Biol. 31 (2011) 1186-1200.

[56] A.G. Baltz, M. Munschauer, B. Schwanhausser, A. Vasile, Y. Murakawa, M. Schueler, N. Youngs, D. Penfold-Brown, K. Drew, M. Milek, E. Wyler, R. Bonneau, M. Selbach, C. Dieterich, M. Landthaler, The mRNA-bound proteome and its global occupancy profile on protein-coding transcripts, Mol. Cell 46 (2012) 674-690.

[57] A. Castello, B. Fischer, K. Eichelbaum, R. Horos, B.M. Beckmann, C. Strein, N.E. Davey, D.T. Humphreys, T. Preiss, L.M. Steinmetz, J. Krijgsveld, M.W. Hentze, Insights into RNA biology from an atlas of mammalian mRNA-binding proteins, Cell 149 (2012) 1393-1406.

[58] S.C. Kwon, H. Yi, K. Eichelbaum, S. Fohr, B. Fischer, K.T. You, A. Castello, J. Krijgsveld M.W. Hentze, V.N. Kim, The RNA-binding protein repertoire of embryonic stem cells, Nat. Struct. Mol. Biol. 20 (2013) 1122-1130. 